\title{
Die hochwürmzeitlichen Rückzugsphasen des Rhein-Vorland- gletschers und der erste alpine Eisrandkomplex im Spätglazial
}

\begin{abstract}
:
The Upper Pleistocene Würm Glaciation Recessional stades of the Alpine foreland glaciers of the Rhine area, and the first Alpine boundary complex of the Late Pleistocene.

In the Rhine area of the Würm glaciation, the recessional stades can be morphologically subdivided by means of glacial boundary complexes. The Würm-Stein-on-the-Rhine complex is evidence for an upper Würm readvance of the foreland glaciers after an intensive melting period $( \pm 16000$ y BP?). The first Late Pleistocene readvance of the local northern Alpine glaciers appears as the Würm-Weissbad complex ( \pm 14000 y BP?), while in the main network of the glaciation no reaction is recognizable. In the Upper Pleistocene of the Würm, the equilibrium line of the Rhine glacier was at an elevation of $1000 \mathrm{~m}$. The first readvance of local glaciers in the Late Pleistocene took place at an elevation of about $1500 \mathrm{~m}$.
\end{abstract}

\section{Key words:}

Upper Pleistocene, Würm glaciation, recessional complexes, equilibrium line altitude, geomorphological maps, deglaciation history.

Alpine foreland (Rhine area), Switzerland N-E, Germany-S

\section{Kurzfassung:}

Im Gebiet des würmzeitlichen Rheingletschers lassen sich die Rückzugsphasen morphologisch mittels Eisrandkomplexen gliedern. Der Komplex Würm-Stein am Rhein belegt einen hochglazialen Wiedervorstoß des Vorlandgletschers nach einer intensiven Abschmelzphase ( \pm 16000 BP?). Ein erster spätglazialer Vorstoß der randalpinen Lokalgletscher zeichnet sich als Komplex Würm-Weißbad ( \pm 14000 BP?) $\mathrm{ab}$, während im Eisstromnetz keine Reaktionen zu erkennen sind. Im Hochglazial liegt die Schneegrenze des Rheingletschers bei 1000 m; der erste spätglaziale Vorstoß der Lokalgletscher erfolgt bei einer Schneegrenze um $1500 \mathrm{~m}$.

\section{Einleitung}

Während des Pleistozäns bedeckten Vorlandgletscher des Rheinsystems wiederholt unterschiedlich große Räume des Molassebeckens zwischen dem Alpenrand und dem Schwäbischen Jura. Die Höhenlage des bündnerisch-vorarlbergischen Alpenraums
- im Mittel um $2000 \mathrm{~m}$ - bewirkte bei absinkender Schneegrenze relativ rasch großflächige Vereisungen, die zunächst noch in Form von Talgletschern ihren Ausgleich fanden. Erst in anhaltenden Hochglazialen mit Schneegrenzen unterhalb $1500 \mathrm{~m}$ vermochte der Rheingletscher sich soweit aufzubauen, $\mathrm{da} ß$ er das Bodenseebecken weitflächig überfahren konnte.

Die vorliegende Arbeit befaßt sich mit Vorgängen während der letzten, der hochwürmzeitlichen alpinen Vergletscherung. Die Vormarschetappen dieser Vereisung liegen noch weitgehend im dunkeln, beginnen aber durch neuere Untersuchungen erste Umrisse anzunehmen (MÜLLER, 1979, 1982; KRAYSS \& KELLER, 1983; KELLER, 1985; KRAYSS, 1985 SCHINDLER, 1985; HIPP, 1986). Demgegenüber liegen zum Stand des Hochwürm-Maximums und zu den Rückzugsphasen schon recht viele Befunde vor, die es gestatten, Gliederungen vorzunehmen und die wichtigsten Abläufe im Zusammenhang darzustellen.

Bereits PENCK erkannte in der grundlegenden Bearbeitung des Rheingletschers (PENCK \& BRÜCKNER, 1909) eine generelle Zweigliedrigkeit der würmzeitlichen Vorlandvereisung, indem er "Äußere» und «Innere» Jungendmoränen unterschied und letztere einem Gletschervorsto $ß$ nach seiner «Laufenschwankung» zuordnete. SCHMIDLE nahm 1914 eine weitere Unterteilung der Eisrandlagen vor, indem er dem "Schaffhausener» und "Stein-Singener» einen "Dießenhofener", "Konstanzer» und "Lindauer» Moränengürtel hinzufügte und diese Stände mittels Isohypsenkarten der Gletscheroberfläche anschaulich darstellte. Seit dem Erscheinen der «Alpen im Eiszeitalter» (PENCK \& BRÜCKNER, 1909) befaßte sich eine größere Zahl von glazialmorphologischen und quartärgeologischen Untersuchungen im Rheingletschergebiet mit vorwiegend regionalen Problemen; einen guten Überblick geben HANTKE (1978-83) und BURGA (1982). Wie die Arbeiten der letzten Jahre zeigen, wird die klassische

Oskar Keller, Dr., Sonderstraße 22, 9034 Eggersriet Edgar Krayss, Myrtenstraße 9, 9010 St. Gallen 


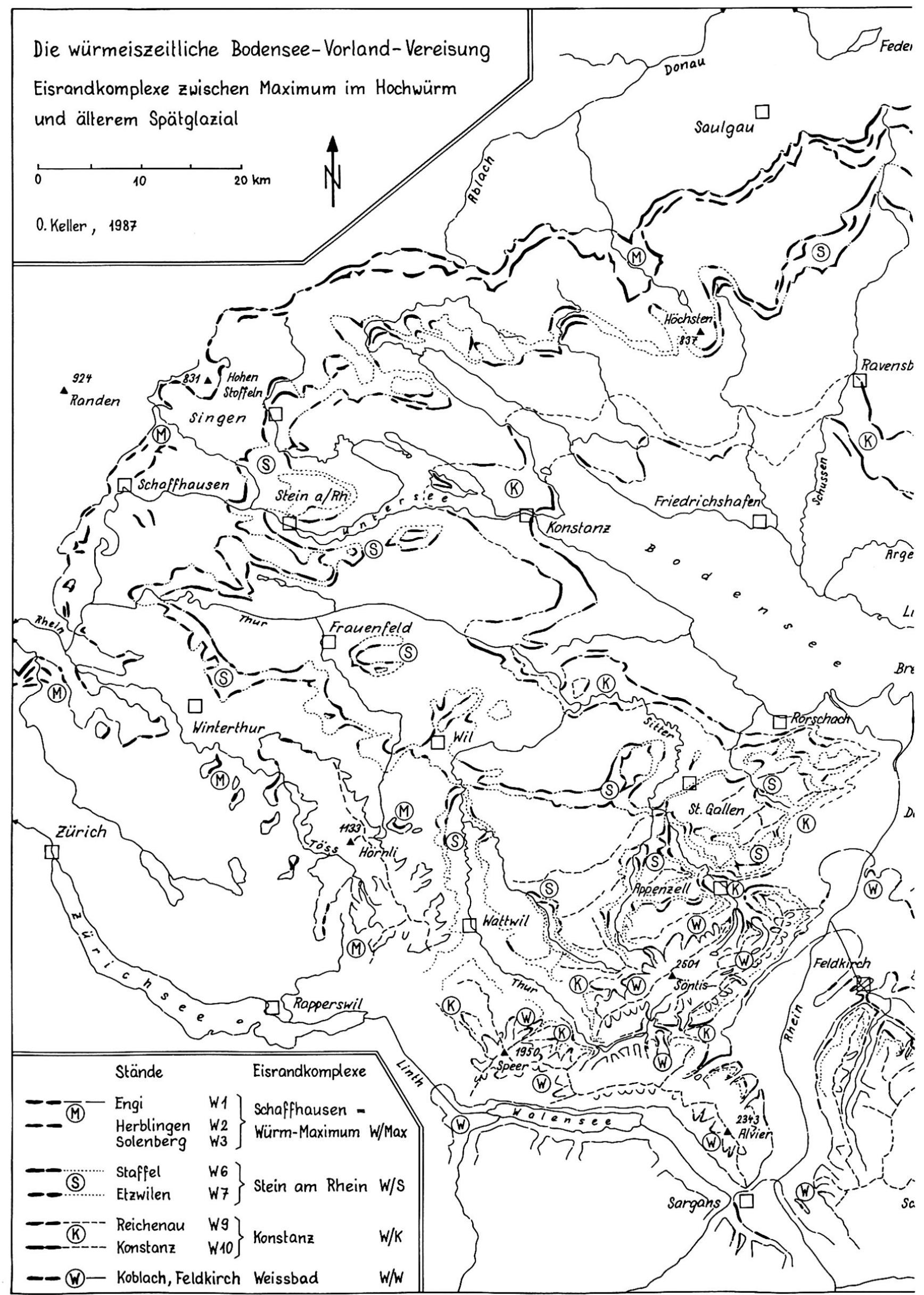

Fig. 1 


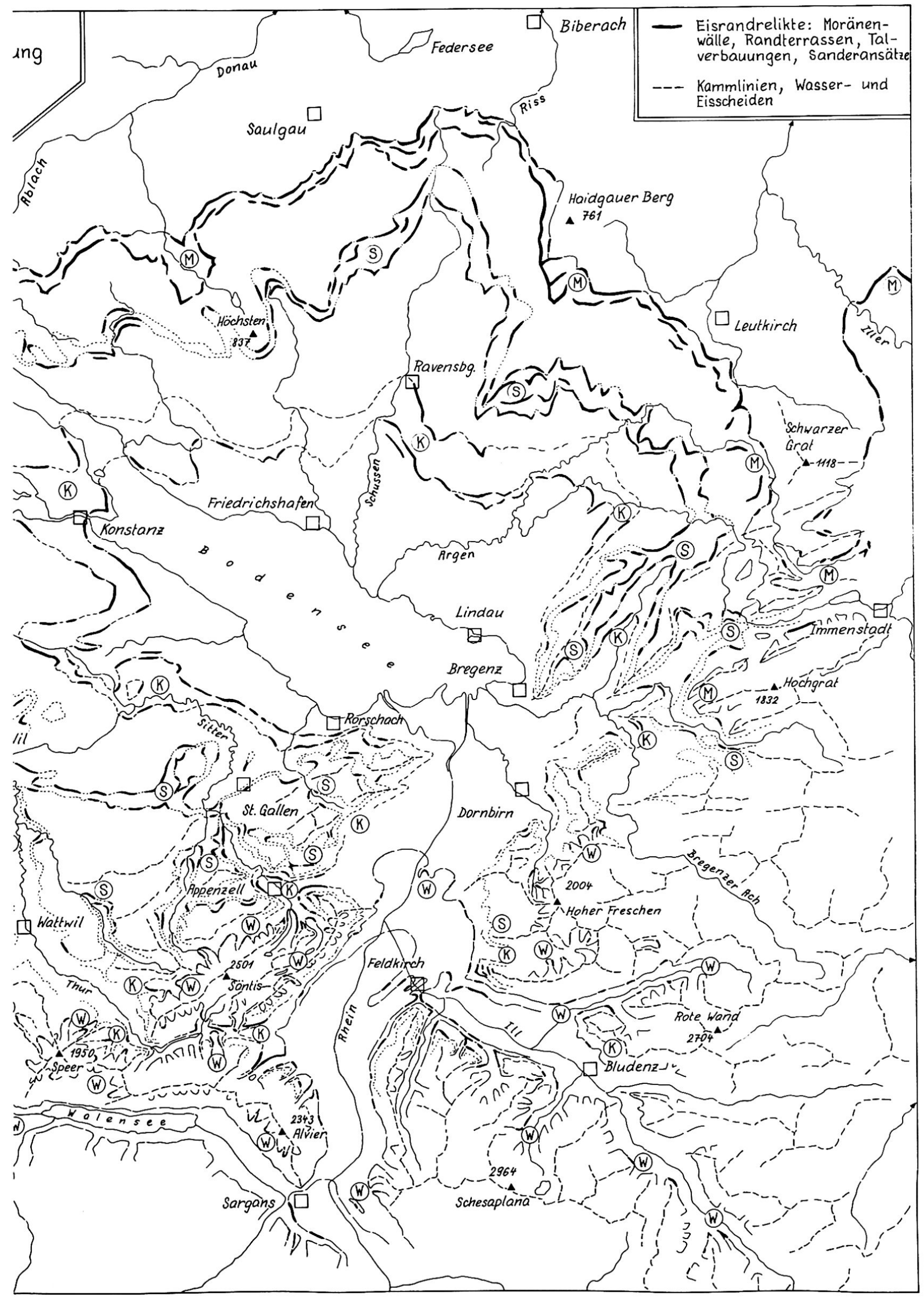


Glazialmorphologie in fruchtbarer Weise durch Sedimentuntersuchungen, Lithostratigraphie und Pollenanalysen ergänzt (W.A. KELLER, 1974; KÄSER, 1980; DE JONG, 1983; SCHINDLER, 1985; HIPP, 1986).

Nach der Abbildung des Bodenseegletschers durch JÄCKLI (1962) im Rahmen einer Vergletscherungskarte der Schweiz erarbeiteten die Autoren erstmals eine detaillierte Gesamtdarstellung einer Bodensee-Vorlandvereisung (KELLER \& KRAYSS, 1980), und zwar für das "Stadial» Würm-Stein am Rhein. Die morphologisch belegbare Klarheit, mit der sich dieser Komplex wie ein Leithorizont durch den ganzen Bodenseeraum hindurch verfolgen läßt, bewog die Autoren damals zur Frage, ob zwischen WürmMaximum und dem «Stadial» Stein am Rhein ein größeres Interstadial vorliegen könnte. Aufgrund neuerer Befunde ist diese Frage indessen zu verneinen.

\section{Die hochwürmzeitlichen Rückzugsphasen}

Wie die Karten (Fig. 1 und 2) zeigen, lassen sich im Bereich des Bodensee-Vorlandgletschers drei Eisrandkomplexe ausgliedern, die noch dem Hochwürm zuzusprechen sind:

- als äußerer der Komplex Schaffhausen = Würmmaximum W/Max

- als mittlerer der Komplex Stein am Rhein W/S

- als innerer der Komplex KonstanzW/K

Unter einem Eisrandkomplex verstehen wir eine mehr oder weniger breite Staffelung von Moränenwällen, Sanderterrassen und Schmelzwasserrinnen, die eindeutig auf einen angrenzenden aktiven Eisrand hinweisen (KELLER \& KRAYSS, 1982). Die Komplexe werden voneinander durch Geländestreifen getrennt, in denen solche Eisrandrelikte weitgehend fehlen. Dafür finden sich in diesen Zwischenzonen verbreitet Seeablagerungen, die auf ein eher flächenhaftes Abschmelzen stagnierender Eiskörper hinweisen. Im Bereich der Zungenbecken-Achsen beträgt die Breite der äußeren Zwischenzone $10-15 \mathrm{~km}$, der innern dagegen $10-40 \mathrm{~km}$.

Der Eisrandkomplex des Wïrm-Maximums W/Max umfaßt die Stände W1 bis W3, eine Numerierung, die sich an die von PENCK (1896) eingeführte und von ERB (1931 u. a.) und SCHREINER (1974 u. a.) übernommene Gliederung der Schotterterrassen und Eisränder im Raum Schaffhausen-Untersee hält.

Die Randlage W1 wurde von den Autoren (KRAYSS \& KELLER , 1983) eingehend beschrieben und kartographisch dargestellt. Sie dürfte während eines relativ kurzfristigen Vorstoßes erreicht worden sein. Ablagerungen sind meist spärlich, während erosive Prozesse überwiegen. Die Stände W2 und W3 sind charakterisiert durch die Bildung mächtiger Sander. die von entsprechend markanten Moränenwällen ausgehen. Im oberschwäbischen Raum erfolgte die Eisrandentwässerung über weite Sanderflächen zur Iller und zur Donau, dazwischen wurden bei Buchau und Wurzach Seen gestaut. Etwas westlich der Ablachrinne lag die kontinentale Wasserscheide zum Rhein. Von hier an floß ein Eisrandstrom zuerst durch enge Täler im Malmkalk des Juras und weiter durch Stauseen im Hegau nach Schaffhausen und schließlich in den Klettgau. Der südliche Randstrom aus derTößrinne fand seinen Weg auf das Rafzerfeld (KRAYSS \& KELLER, 1982).

Aus der Zeit der ersten großen Abschmelzphase erkennen wir die Eisrandlagen W4 und W5: wir finden ihre Spuren in der äußeren Zwischenzone W/Max$W / S$. In Oberschwaben blieb zwischen Isny und Pfullendorf die Entwässerung zur Donau noch erhalten: der Abfluß erfolgte jetzt vorwiegend über flache Seen und Durchbrüche im äußeren Moränenkranz: Begünstigt durch das starke Gefälle zum Rhein setzte in den Hangzonen zwischen Stockach und Engen intensive Erosion unter Bildung von Schmelzwasserrinnen ein. Dem gleichen Vorgang begegnen wir in noch ausgeprägterem Maße am Südrand der Vorlandvergletscherung, wo sich die markanten Schmelzwassertäler vom Rindal bis zum Tößtal in die Molasse einzutiefen begannen (KELLER, 1976). Größere Seen lagen hinter den Stauschwellen der Moränenwälle und Sander, so bei Pfungen, Feuerthalen und Thayngen. Im Hegau bildeten sich Eisstauseen. Die äußere Zwischenzone wird somit weitgehend von Erosionsformen und Stillwassersedimenten geprägt.

Der Eisrandkomplex Stein am Rhein W/S - PENCKS Kranz der Innern Jungendmoränen - belegt eindeutig ein Wiedererstarken des hochwürmzeitlichen glazialen Regimes. Bereits im äußeren StandW6 begann sich der abschmelzende Eiskörper zu stabilisieren (KELLER \& KRAYSS, 1980). Klare Merkmale eines Gletschervorstoßes trägt jedoch der StandW7, wobei die Schwankungsbreite des Wiedervorstoßes noch unbestimmt ist. Charakteristisch für den Stand W7 ist eine Abfolge markanter Moränenwälle mit anschließenden Sandern, die vor allem im Bereich der rheinwärts gerichteten Zungenbecken sehr ausgeprägt ist.

In Oberschwaben entwässerte sich nur noch der Schussenlappen zur Donau. Bedingt durch dasTrokkenfallen des Überlaufs zur Iller, mußte sich der nordöstliche Eisrandstrom einen Weg über die Wolfegger Rinne zur Riß und zur Donau bahnen. Bei Singen, Dießenhofen und Winterhur überschotterten die Schmelzwasserströme die feinkörnig verfüllten Seen, während sich von St. Gallen an eine Abfolge von Sandern bis zurTöss entwickelte. Weiträumige glaziale Stauchungen in den Schottern des Aadorfer Feldes belegen permafrostartige Verhältnisse am Gletscherrand (sCHINDLER et al., 1978) und damit die hochglaziale Natur des Komplexes W/S. 


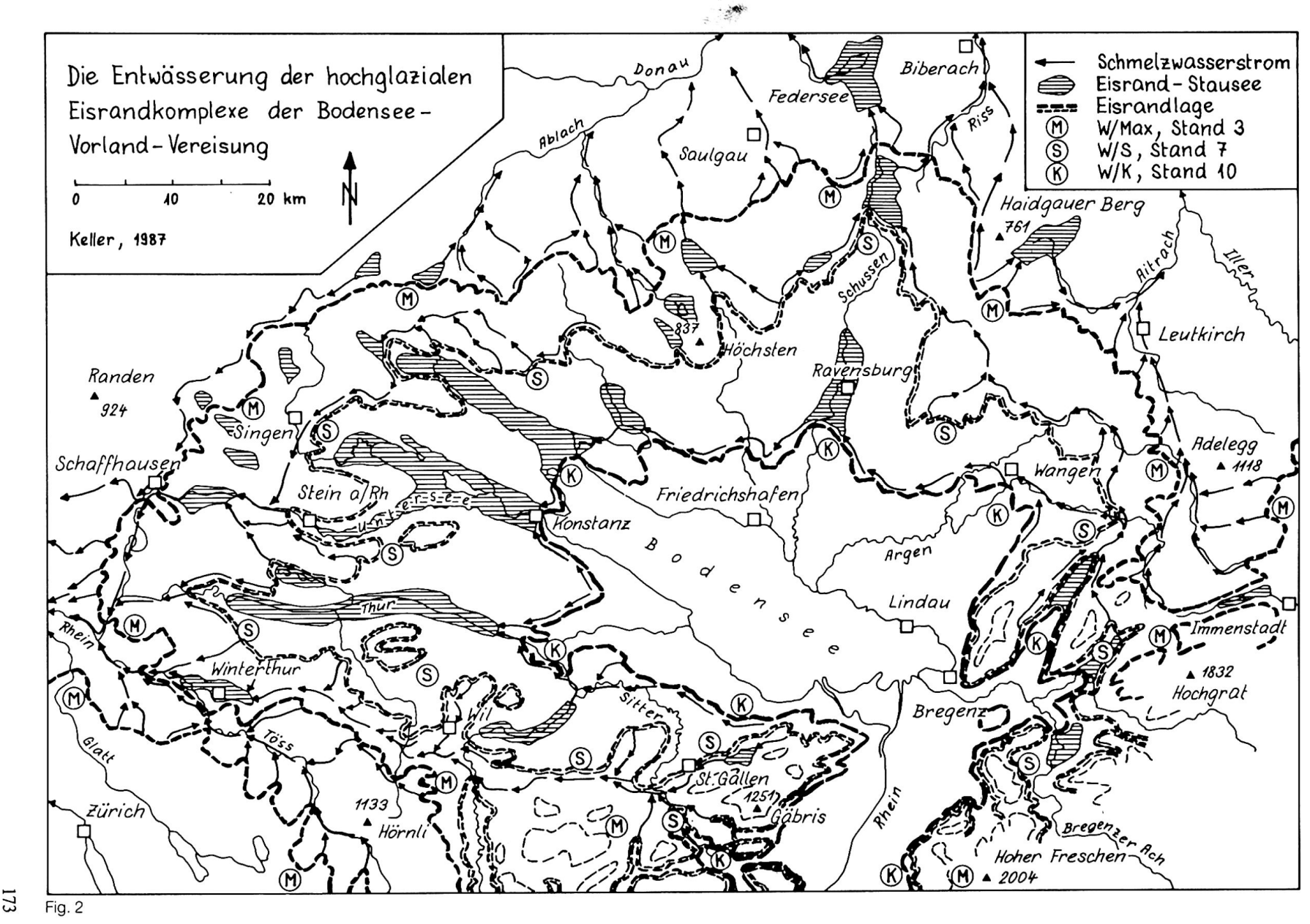


Der Umschwung im Gletscherregime, der mit dem Rückschmelzen vom Stand W7 seinen Anfang nahm, muß radikal gewesen sein. In den meisten Flußtälern finden wir unmittelbar anschließend an die Endmoränen des Standes W7 mächtige feinkörnige Seeablagerungen. In der ganzen innern Zwischenzone W/S-W/K sind Hinweise auf Eisrandlagen sporadisch und kaum mehr als von lokaler Bedeutung. Zusammenhängende Wälle, Schmelzwasserrinnen und Kamesterrassen treten beckeneinwärts erst wieder in einem Geländestreifen auf, der auf der Karte (Fig. 1) als Eisrandkomplex Konstanz $W / K$ dargestellt ist.

Im Stand W10, der einigermaßen deutlich erfaßbar ist, stirnte der Gletscher am untern Ende des Bodensees. Die nordseitige Eisrandentwässerung erfolgte durch frisch eingetiefte Rinnen und Seen bei Wangen, Ravensburg und Markdorf zum Überlingersee. Auf der Südseite flossen Sitter und Thur als Vorfluter bereits in ihren heutigen Tälern. Die Vorgänge des Eisabbaus während dieser Übergangsphase vom Hoch- zum Spätglazial sind zurzeit noch Gegenstand eines Forschungsprojektes, das die Autoren gemeinsam mit Prof. G. Furrer, Zürich, bearbeiten. Morphologische Argumente sprechen vorläufig dafür, daß wir es beim Komplex W/K mit einer Stabilisierung des zurückschmelzenden Vorlandgletschers zu tun haben.

\section{Der Eisrandkomplex Würm-Weißbad}

Während die Staffeln des Konstanzer Eisrandkomplexes noch großzügig das zentrale Becken des Bodensees umgürten, liegt der Hauptstand des nächst interneren Komplexes von Weißbad-Koblach bereits weit zurück im mittleren Rheintal, eingespannt zwischen die Gebirgssporne des Alpentors von Oberriet-Götzis. Zwar beachtete schon PENCK (1909) die zugehörigen Serien von Randrinnen und Terrassen auf der Ostseite des Rheintals zwischen Feldkirch und Hohenems, aber erst HANTKE (1970) postulierte ein Feldkirch-Stadium mit mehreren Ständen. Eigene Untersuchungen (KELLER, 1983-86, Arbeit im Druck) haben nun gezeigt, daß in der Tat $N$ Feldkirch ein Eisrandkomplex vorliegt, dessen Hauptstand aber bei Koblach anzusetzen ist. Im Gegensatz zu denjenigen im Vorlandbecken sind jedoch die Eisrandlagen hier verwischter und vor allem nur in Bruchstücken erhalten.

Wendet man sich nun der synchron laufenden Lokalvergletscherung des Alpsteins (Säntisgebirge), des Speers, des Alviers oder des Hohen Freschen und des Laternsertales zu, so sind die zu W/K äquivalenten Eisrandrelikte generell weniger deutlich als der durch besondere Frischheit auffallende Komplex beim Weißbad südlich Appenzell (Fig. 3), der mit Koblach zu parallelisieren ist. Bereits PENCK (1909,
S. 438) erwähnt diesen Gletscherstand und ordnet ihn seinem «Bühl-Stadium» zu. Da hier im weiten Umkreis und vor allem gegenüber dem Rheintal die auffälligsten Eisrandzeugen auftreten, soll dieser älteste spätglaziale und erste alpine Eisrandkomplex mit «Weißbad» =W/W bezeichnet werden .

Entlang der Alpenrandzone, in der die Lokalgletscher bereits selbständig waren, ergibt derWeißbadKomplex insofern einen weiteren glazialmorphologischen Leithorizont, als hier mit scharfer Grenze nach außen kantige, frische Wallformen, aufgebaut aus Blockmoräne und flächenhaft übersät mit dichter erratischer Blockstreu, einsetzen. Dies gilt jedoch nicht für die «Riesen», nämlich den Rheinund den Illgletscher, die noch dem inneralpinen Eisstromnetz angehören und bei Gletscherlängen von $70-150 \mathrm{~km}$ aus der Ferne ernährt werden.

Während Formen und Sedimentsstrukturen der nur wenige bis $10 \mathrm{~km}$ langen lokalen Eisströme einen Vorsto $ß$ ausweisen, belegen die Eisrandgebilde der großen Hauptgletscher nur einen Stillstand während des allgemeinen Rückschmelzens. Der markante Vormarsch der Lokalgletscher erfordert eine einschneidende Klimaverschlechterung, die aber kurzfristig im Jahrhundertbereich erfolgt sein muß da das Eisstromnetz nicht oder nur in geringem Ausmaß zu reagieren vermochte.

Die Berechnung der basalen Schubspannung liefert für 20 verschiedenartige W/W-Lokalgletscher Werte von 1-1,5 bar, welche auf sich aufbauende, sehr aktive Gletscher schließen lassen. Für die drei Haupttalgletscher (Rhein-Bodensee, Rhein-Walensee, III) ergeben sich lediglich Schubspannungen um 0,5 bar, was für stagnierende oder sich kaum bewegende Eismassen charakteristisch ist. Dieser Befund stützt die Vorstellung einer kurzfristigen, aber doch eingreifenden Klimaveränderung.

\section{Glaziologie, Klima und Chronologie}

Seit nun die würmzeitlichen Eisrandkomplexe im gesamten Rheingletscherareal rekonstruiert sind (KELLER, KRAYSS, 1980, 1983, Arb. im Druck), können die Isohypsenbilder der inneralpinen Eisstromnetze durch Extrapolieren gewonnen werden. Aus den darauf basierenden hypsographischen Kurven (Fig. 4) lassen sich eine Reihe glazio- und klimatologischer Folgerungen ableiten.

Die Kurvenformen charakterisieren den Gletschertyp. Der hinter dem Stirnbereich mäßig steigende Kurvenabschnitt widerspiegelt die Vorlandvereisung mit großer Ausdehnung bei schwachem Gefälle der Eisoberfläche. Das steile Mittelstück entspricht der Auslaßkanalzone im Rhein- und Walenseetal mit geringen Flächenanteilen pro Höhenstufe. Im oberen, wieder flacheren Abschnitt lassen sich die hochgelegenen, weiten Firnareale erkennen. Die im W/W fehlende Vorlandausbreitung ma- 


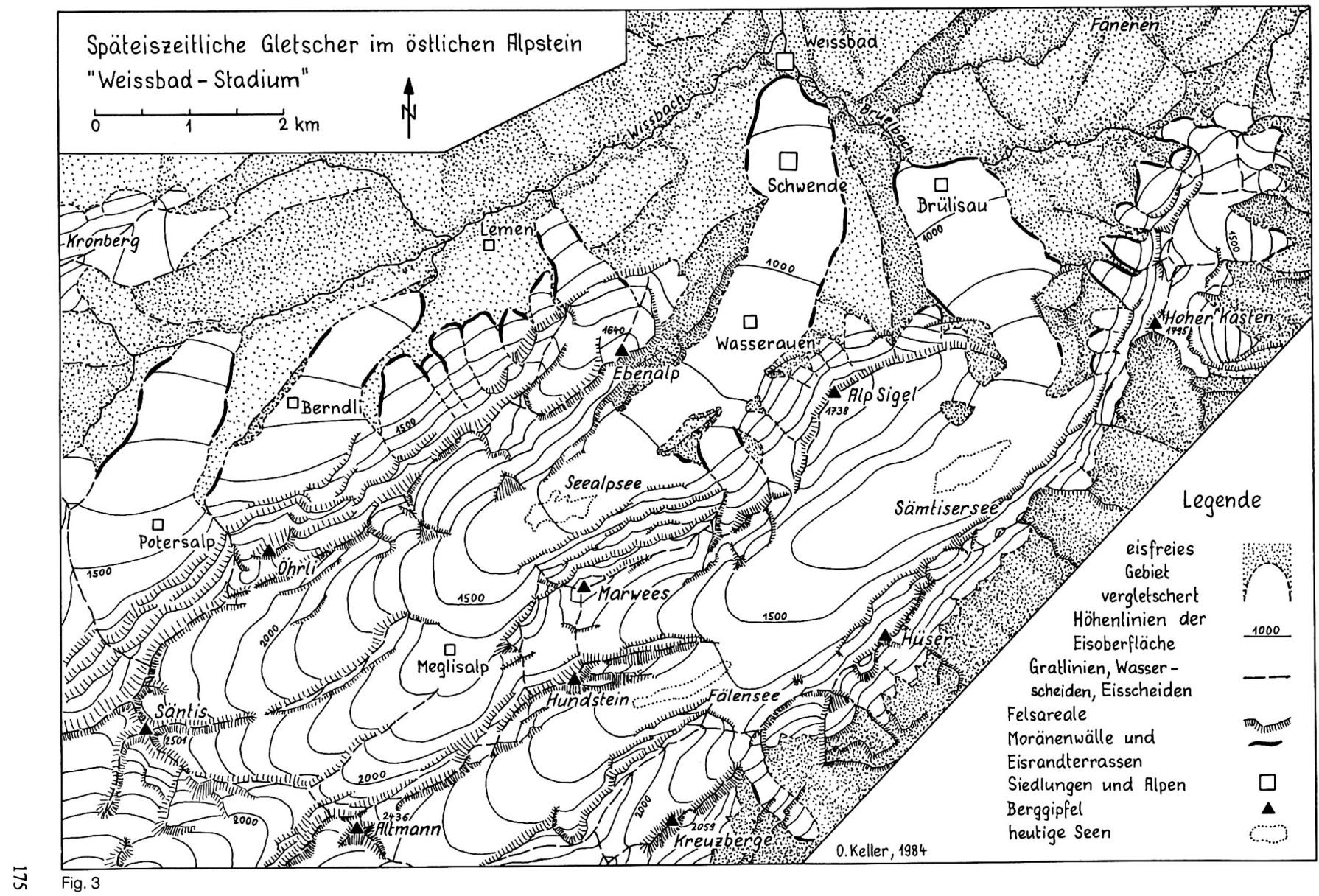


Hypsographische Kurven und Schneegrenzen

des Rhein-Linth-Gletschersystems

(1) $R / B$ Riss-Biberach

(2) W/Max Würm-Maximum, stand 1

(3) W/S Würm-Stein am Rhein, Stand 6

(4) $W / K \quad W u ̈ r m-K o n s t a n z$, Stand 10

(5) W/W Würm-Weissbad, Stand Koblach

(6) $S_{t} V$ Terrainflächen des Vorlandbeckens W/Max

(7) $S_{t} B$ Terrainflächen des rheinischen Bündnerlandes

申 Schneegrenzenlage bei AAR 0,67

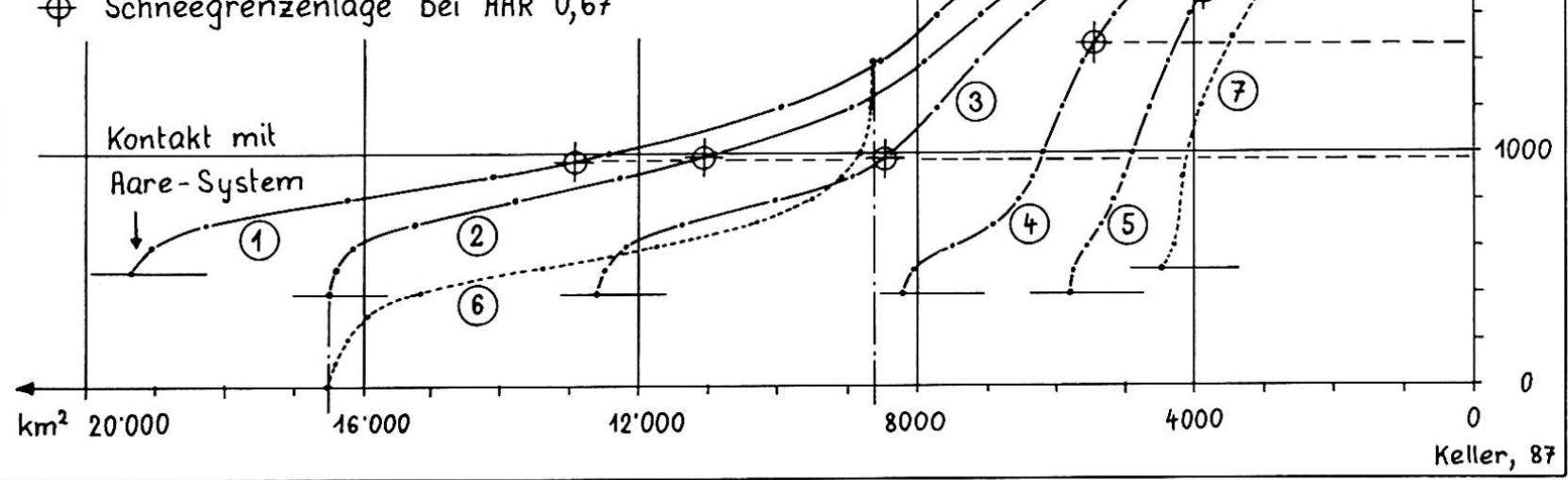

Fig. 4

nifestiert sich auch in der Kurve, indem die untere Verflachung ausfällt. Dieser Kurvenverlauf ist typisch für einen Talgletscher mit ausgedehnten Firnbereichen und langgestreckter, schmaler Zunge in Tallage. Nicht nur im Kartenbild (Fig. 1), sondern auch an der hypsographischen Kurve zeigt sich die geringe Vorlandausbreitung des Konstanzer Komplexes, der damit den Übergang vom Vorland- zum alpinen Talgletscher herstellt.

Die Kurve der Terrainflächen des Vorlandbeckens (Fig. 4) bezieht sich auf das vor dem Alpenrand gelegene, im W/Max vergletscherte Gebiet des Bodensee- und des Zürichsee-Glattal-Beckens, wobei die Tal- und Trogfüllungen ausgeräumt gedacht sind. Der Vergleich dieser Kurve mit derjenigen der W/Max-Eisoberfläche liefert für das Vorland eine mittlere Eismächtigkeit von $355 \mathrm{~m}$, woraus bei $7900 \mathrm{~km}^{2}$ vergletschertem Areal ein Eisvolumen von $2800 \mathrm{~km}^{3}$ resultiert. Die aus dem Eiskuchen aufragenden Nunataker werden durch die über die Vereisungskurve bis auf $1400 \mathrm{~m}$ aufstrebende Spitze der Terrainkurve wiedergegeben.

Aus der Höhenlage der Schneegrenzen lassen sich erste Hinweise auf das Klima ableiten. Für alpine Gletscher kann mit genügender Genauigkeit die Schneegrenze mit der rechnerisch ermittelten Flächenteilungsgrenze $S_{c}: S=0,67=$ AAR gleichgesetzt werden (GROSS, KERSCHNER und PATZELT, 1978). Dabei bedeuten $S_{c}=$ Akkumulationsareal,
$\mathrm{S}=$ gesamte Gletscherfläche, AAR = Accumulation Area Ratio. Inwieweit der AAR $=0,67$ auch für hochglaziale Vorlandgletscher angewendet werden kann, ist unsicher, da die den AAR beeinflussenden Niederschlagsverhältnisse nicht bekannt sind.

Während den Vorlandvereisungen lag bei einem AAR $=0,67$ die Sgr (Schneegrenze) mit knapp 1000 $\mathrm{m}$ ü. M. sehr tief und noch auf dem Vorlandeiskörper (Fig. 4). Besonders auffällig ist dabei, daß sowohl im W/Max als im W/S, das heißt bei deutlich verschiedenen Gletscherausmaßen, die Sgr gleiche Höhen aufwies. Das dürfte kein Zufall sein, da auch im rißzeitlichen Stand Biberach (Fig. 4) beim nochmals wesentlich größeren Rheingletscher sich wieder dieselbe Höhenlage einstellte. Verschiedene Modelle sind möglich: Es lagen in allen 3 Phasen ähnlich tiefe Temperaturen vor, die aber verschieden lang andauerten und damit die Eismassen ungleich mächtig anwachsen ließen; oder das Klima war im Riß besonders trocken (AAR 0,75, Sgr um $900 \mathrm{~m}$ ) und $\mathrm{im} \mathrm{W} / \mathrm{S}$ gegenüber dem W/Max bereits ozeanisch getönt (AAR 0,6, Sgr um $1100 \mathrm{~m}$ ). Für die Sgr-Höhenlage sind die Sommertemperaturen mit ca. $+5^{\circ} \mathrm{C}$ im Juli maßgebend (MESSERLI, 1967 und weitere Autoren), was im W/Max (Fig. 4) der Höhe 1000 m ü. M. entspricht. Die heutige Sgr findet sich am Säntis bei ausgeschaltetem Expositionseinfluß auf $2500 \mathrm{~m}$ ü. M., wo Julitemperaturen von 


\begin{tabular}{|c|c|c|c|}
\hline \multicolumn{4}{|c|}{ Hoch- und Spätwürm - Gliederung } \\
\hline \begin{tabular}{l|} 
Zeit- \\
räume
\end{tabular} & $\begin{array}{l}\text { Kaltphasen, Stadien, } \\
\text { Eisrandkomplexe }\end{array}$ & $\begin{array}{c}\left.10^{3}\right] \\
B P\end{array}$ & $\begin{array}{l}\text { Warmphasen, } \\
\text { Interstadiale }\end{array}$ \\
\hline $\begin{array}{l}\text { Post- } \\
\text { glazial }\end{array}$ & & $-10-$ & Präboreal \\
\hline 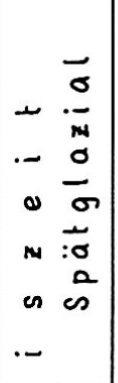 & $\begin{array}{l}\text { Egesen } \\
\text { Daun? } \\
\text { Daun?, Clavadel, } \\
\text { Gschnitz, Steinach } \\
\text { Sargans, Feldkirch } \\
\text { Weissbad-Koblach }=W / W\end{array}$ & $-12-$ & $\begin{array}{l}\text { Flleröd } \\
\text { Bölling } \\
\text { Interstadial? }\end{array}$ \\
\hline $\begin{array}{cc}\omega & - \\
E & \sigma \\
= & \frac{\sigma}{N} \\
= & \frac{\sigma}{\sigma} \\
= & \frac{\sigma}{0} \\
0 \\
x\end{array}$ & $\begin{array}{l}\text { Konstanz }=W / K \\
\text { Stein am Rhein }=W / S \\
\text { Schaffhausen }=W / \text { Max }\end{array}$ & $-\begin{array}{r}- \\
-16- \\
-18 \\
- \\
-18\end{array}$ & Interstadial? \\
\hline & & & Keller, 87 \\
\hline
\end{tabular}

Fig. 5

$+5,6^{\circ} \mathrm{C}$ herrschen. Aus der Höhendifferenz läßt sich auf eine hochglaziale Temperaturdepression von $7-8^{\circ}$ gegenüber heute schließen, die bei kalttrockenem Klima und deshalb tieferer Sgr sogar $10^{\circ} \mathrm{C}$ ausmachen könnte.

Der sprunghafte Anstieg der Sgr auf $1500 \mathrm{~m}$ im W/K und $1700 \mathrm{~m}$ im W/W belegt den deutlichen Temperaturanstieg, der nach dem eigentlichen Hochglazial den Vorlandgletscher zurückschmelzen ließ. Da sich der AAR 0,67 auf einen Gletscher im Gleichgewicht bezieht, kann während den Abschmelzphasen nach W/S die Sgr temporär durchaus gegen $2000 \mathrm{~m}$ angestiegen sein.

Wie der Klimaverlauf ist auch die Chronologie des Zeitabschnitts vom Würm-Hochglazial bis zum Spätglazial erst vage bestimmbar. Ein leidiger Widerspruch besteht z. B. zwischen den ${ }^{1+} \mathrm{C}$-Datierungen von GEYH \& SCHREINER (1984), die den gesamten Eisrückzug von W/Max bis W/K in den Zeitraum von 14800 bis $14100 \mathrm{BP}$ setzen, und den Untersuchungen von LISTER et al. (1984), wonach der untere Zürichsee - also eine Situation post W/S - schon vor 15000 BP eisfrei wurde. Die in Fig. 5 aufgrund von ${ }^{1+} \mathrm{C}$-Daten und Pollenspektren, aber auch im Vergleich mit überregionalen Erkenntnissen erwogene Ordnung kann zwar als problematisch, aber doch einigermaßen wahrscheinlich angesehen werden.

\section{Literatur}

BURGA, C. A. (1982): Zur Erforschung des Quartärs in der Schweiz. Geogr. Helv. 1982/2: 68-74.

ERB, L. (1931): Erläuterungen zu Blatt Hilzingen. Geol. Spezialkarte von Baden. Freiburg.

GEYH, M. A. u. SCHREINER, A. (1984): ${ }^{14} \mathrm{C}$-Datierungen an Knochen- und Stoßzahnfragmenten aus würmeiszeitlichen Ablagerungen im westlichen Rheingletschergebiet (BadenWürttemberg). Eiszeitalter und Gegenwart 34: 155-161.

GROSS, G., KERSCHNER, H. u. PATZELT, G. (1978): Methodische Untersuchungen über die Schneegrenze in alpinen Gletschergebieten. Zeitschr. für Gletscherkde. und Glazialgeol. XII/2: 223-251.

HANTKE, R. (1970): Zur Diffluenz des würmeiszeitlichen Rheingletschers bei Sargans und die spätglazialen Gletscherstände in der Walensee-Talung und im Rheintal. Vjschr. Natf. Ges. Zürich 115/1: 101-126.

HANTKE, R. (1978), (1980), (1983): Eiszeitalter. Ott, Thun. Bde. 1, 2 u. 3.

HIPP, R. (1986): Zur Landschaftsgeschichte der Region Bischofszell. Eine glazialmorphologische Arbeit. Mitt. Thurg. Natf. Ges. 47: 5-117.

JÄCKLI, H. (1962): Die Vergletscherung der Schweiz im Würmmaximum. Eclogae geol. Helv. 55/2: 285-294.

JONG, M. G. D. DE (1983): Quaternary deposits and landforms of western Allgäu (Germany) and the deglaciation after the last major pleistocene ice advance. Thesis Amsterdam: $186 \mathrm{~S}$.

KÄSER, U. J. (1980): Glazialmorphologische Untersuchungen zwischen Töß und Thur. Diss. Univ. Zürich. Juris, Zürich: $135 \mathrm{~S}$.

KELLER, O. (1974): Untersuchungen zur Glazialmorphologie des Neckertales (Nordostschweizer Voralpen). Jb. St. Gall. Natw. Ges. 80: 202 S.

KELLLER, O. (1976): Das Rindal; zur Genese eines Urstromtales in der NE-Schweiz. Geogr. Helv. 1976/4: 161-166.

KELLER, O. (1985): Zum Eisaufbau des hochwürmzeitlichen Rheingletschers im Bereich des Alpenrandes. Phys. Geogr. Vol. 16. Zürich: 31-42.

KELLER, O. U. KRAYSS, E. (1980): Die letzte Vorlandvereisung in der Nordostschweiz und im Bodensee-Raum (Stadialer Komplex Würm-Stein am Rhein). Eclogae geol. Helv. 73/3: 823-838.

KELLER, O. u. KRAYSS, E. (1982): Methoden zur Rekonstruktion eiszeitlicher Vorland- und Talgletscher. Phys. Geogr. Vol. 1, Zürich: 127-136.

KELLER, W. A. (1977): Die Rafzerfeldschotter und inre Bedeutung für die Morphogenese des zürcherischen Hochrheingebietes. Diss. Univ. Zürich. Vjschr. Natf. Ges. Zürich 122/3: 357-412.

KRAYSS, E. (1985): Zur Gliederung des Eisaufbaus der hochwürmzeitlichen Bodensee-Vorlandvergletscherung. Phys. Geogr. Vol. 16, Zürich: 23-30.

KRAYSS, E. u. KELLER, O. (1982): Zur Paläogeographie der Tößrinne im Würm-Hochglazial. Phys. Geogr. Vol. 1, Zürich: 205-214.

KRAYSS, E. u. KELLER, O. (1983): Die Bodensee-Vorlandvereisung während des Würm-Hochglazials. Schriften Verein für Gesch. des Bodensees Bd. 101. Friedrichshafen 7: 113-129. 
LISTER, G. u. KELTS, K. et. al. (1984): Correlation of paleoclimatic record in lacustrine sediment sequences: ${ }^{14} \mathrm{C}$ dating by AMS. Nuclear Inst. and Methods in Physics Res. B 5: 389-393.

MESSERLI, B. (1967): Die eiszeitliche und gegenwärtige Vergletscherung im Mittelmeerraum. Geogr. Helv. 22: 105-228.

MÜLLER, E. (1979): Die Vergletscherung des Kt. Thurgau während der wichtigsten Phasen der letzten Eiszeit. Mitt. Thurg. Natf. Ges. 43: 47-73.

MÜLLER, E. (1982): Eisaufbauprozeß des würmzeitlichen Rheingletschers in der Ostschweiz. Phys. Geogr. Vol. 1, Zürich: 221-230.

PENCK, A. (1986): Die Glazialbildungen um Schaffhausen. In: NÜESCH, J.: Das Schweizersbild. N. Denkschr. allg. schweiz. Ges. ges. Naturw. 35
PENCK, A. u. BRÜCKNER, E. (1909): Die Alpen im Eiszeitalter. Tauchnitz, Leipzig. 3 Bde.

SCHINDLER, C. (1985): Geologisch-geotechnische Verhältnisse in Schaffhausen und Umgebung. Beitr. Geol. Schweiz, Kleinere Mitt. 74: $119 \mathrm{~S}$.

SCHINDLER, C., RÖTHLISBERGER, H. u. GYGER, M. (1978): Glaziale Stauchungen in den Niederterrassen-Schottern des Aadorfer Feldes und ihre Deutung. Eclogae geol. Helv. 71: 159-174.

SCHMIDLE, W. (1974): Die diluviale Geologie der Bodenseegegend. Westermann, Braunschweig, Berlin: $113 \mathrm{~S}$.

SCHREINER, A. (1974): Erläuterungen zur Geologischen Karte des Landkreises Konstanz mit Umgebung 1:50 000. Geol. Landesamt Baden-Württemberg: $286 \mathrm{~S}$. 